\title{
Modelling of Malaria Transmission Using Delay Differential Equation
}

\author{
Kipkirui Mibei $^{1}$, Kirui Wesley ${ }^{2}$, Adicka Daniel ${ }^{1}$ \\ ${ }^{1}$ Department of Mathematics, Faculty of Science and Technology, University of Kabianga, Kericho, Kenya \\ ${ }^{2}$ Department of Mathematics, Faculty of Science South Eastern, Kenya University, Kitui, Kenya
}

Email address:

kipkiruimibei@yahoo.com (K. Mibei), kirwes@seku.ac.ke (K. Wesley), adickadaniel78@yahoo.com (A. Daniel)

${ }^{*}$ Corresponding author

\section{To cite this article:}

Kipkirui Mibei, Kirui Wesley, Adicka Daniel. Modelling of Malaria Transmission Using Delay Differential Equation. Mathematical Modelling and Applications. Vol. 5, No. 3, 2020, pp. 167-175. doi: 10.11648/j.mma.20200503.15

Received: May 10, 2020; Accepted: July 14, 2020; Published: August 4, 2020

\begin{abstract}
Malaria is one of the major causes of deaths and ill health in endemic regions of sub-Saharan Africa and beyond despite efforts made to prevent and control its spread. Epidemiological models on how malaria is spread have made a substantial contribution on the understanding of disease changing aspects. Previous researchers have used Susceptible Exposed-Infectious-Recovered (SEIR) model to explain how malaria is spread using ordinary differential equations. In this paper we develop mathematical SEIR model to define the dynamics of the spread of malaria using Delay differential equations with four control measures such as long lasting treated insecticides bed nets, intermittent preventive treatment of malaria in pregnant women (IPTP), intermittent preventive treatment of malaria in infancy (IPTI) and indoor residual spraying. The model is analyzed and reproduction number derived using next generation matrix method and its stability is checked by Jacobean matrix. Positivity of solutions and boundedness of the model is proved. We show that the disease free equilibrium is locally asymptotically stable if $\mathrm{R}_{0}<1\left(\mathrm{R}_{0}\right.$ - reproduction number) and is unstable if $\mathrm{R}_{0}>1$. Numerical simulation shows that, with proper treatment and control measures put in place the disease is controlled.
\end{abstract}

Keywords: Stability, Basic Reproduction Number, Delay Differential Equations

\section{Introduction}

Malaria is one of the most pandemic disease that remains arguably the greatest threat in our society and has remained the main cause of deaths in Africa and many regions of the world. Malaria was a major bottleneck in military camps in the United States where they initiated malaria campaigns to control the menace. In 2015 World Health organization (WHO) estimate on the cases of malaria to be 214 million resulting in 438,000 deaths, majority of these were from Africa. Sub-Saharan Africa continues to exhibit a considerably high number of epidemics of malaria which results to many deaths. Furthermore, WHO (2016) estimates that there were 216 million quantifiable cases of malaria and 445,000 people perished of whom 306,000 were children under 5 years and were mainly from Africa $[1,2]$.

Malaria is transmitted by Plasmodium parasite. One gets malaria by being bitten by infected female anopheles mosquito. The mosquito must have been infected from blood meal of infected persons. Blood of infected person has microscopic malarial parasite that can be passed onto a mosquito when it bites such an individual. The malarial parasite incubates for about seven days after which it becomes infectious and if a mosquito bites a new individual again the parasite from the blood meal will mix with mosquito's saliva and can be transmittable to the person being bitten. Malaria symptoms appears within 9-14 days. The most common symptoms are headache, fever and vomiting. Other ways through which malaria can be spread is by blood transfusion or sharing used needles or syringes of the blood which is contaminated. Also delivery or before delivery of the new born baby, the mother may pass over the disease to the baby. Severe malaria can lead to cerebral malaria, which is associated with unconsciousness, seizures, or other neurologic anomalies. Risks associated with malaria in expectant mothers include maternal anaemia, low weight in infants, immature delivery and increased infant and 
maternal deaths [14]. The prevalence of malaria has been on the rise owing to malaria parasite developing resistance to drugs, mosquito-insecticide resistance and weak malaria intervention measures $[3,9]$. This therefore warrants efficient and effective control measures on the spread of malaria through mathematical modelling. SEIR differential model for humans and SEI for mosquitoes was developed to study the dynamics of spread of malaria and incorporate Preventive measures. For instance, intermittent preventive treatment of malaria in pregnant mothers, Long- Lasting Insecticides Treated Nets (LLINS), indoor residual spraying (IRS).

\section{Review of Related Literature}

Jessica [7] studied malaria spread dynamics for humans and mosquito populations by considering vectorial transmission, vertical transmission of disease and a force of infection which measure the influence that occurs in the disease transmission rate which an infected human is introduced into mosquito population. The study examine a SEIR model for humans and SIR model for mosquitoes and fail to incorporate preventive and control measures to reduce malaria prevalence. In the analysis revealed the existence of three steady states, the disease free equilibrium and two endemic equilibrium and that when $R_{o}<1$, then disease is controlled and when $R_{o}>1$, the disease persists. In the study ordinary differential equations were used, which in this paper is addressed by introducing delay differential equations to cater for latency period that take place between when a mosquito bites and human becoming infected.

Sunita [5], Studied SEIR model for human and SI model for mosquito population. SEIR model took into account new immigrants in the population who are susceptible, exposed and infective.

Impressed by Sunita's work [5], Nisha [12] analysed the steadiness of SEIR model for malaria with infectious migrants but failed to carry out simulation and sensitivity analysis of the given model which was necessary so as to understand the effect of infective immigrants on the spread of malaria in a population. Similar studies were carried by Mojeeb [9] who used a SEIR mathematical model using ordinary differential equations with four control measures such as reducing contact rate between human and mosquito's, reducing the infection rate between humans, use of active malaria drugs and treated mosquito nets.

Ephraim [10] studied the dynamics of several species and strains of malaria. In the model analyzed four species of the malaria parasite and found out that some species of the parasite have evolved into strains that are resistant to treatment, he made assumption that there was no immunity to disease. The model found out that all species or strains persist for some time for the reproduction number greater than one, however the species or strain with the highest reproduction number eventually displace the others. In the model did not consider factors such as seasonality, age structure of humans and mosquitoes' incubation period and spatial distribution.
From the above literature malaria transmission was modelled using ordinary differential equation. In this paper we have modelled the spread of malaria using delay differential equations because of time lags between when a mosquito bites and one becoming sick. We have incorporated four control measures so as to control the spread.

\subsection{The Method of Solution}

In this section we formulate the model, generate the model equations, and find the reproduction number and study existence of disease free equilibrium and its stability.

\subsection{The Model}

In this model, the variables $\mathrm{h}$ and $\mathrm{m}$ denotes humans and mosquitoes population respectively and $t$ is time.

The SEIRS model is used to develop human population and the sum of the entire population is given as:

$$
N_{h}=S_{h}+E_{h}+I_{h}+R_{h}
$$

Where; subscripts h-represents human population

$\mathrm{N}_{\mathrm{h}}$ - total human population

$S_{h}$-susceptible humans

$E_{h}$-exposed humans

$I_{h}$-infectious humans

$R_{h}$-recovered humans respectively.

Similarly, Susceptible-Exposed-Infected (SEI) model is used to develop mosquito population and the Sum total of population is given as;

$$
N_{m}=S_{m}+E_{m}+I_{m}
$$

Where; subscript m-represents mosquito population

$\mathrm{N}_{\mathrm{m}}$ - total mosquito population

$S_{m}$-susceptible mosquitoes

$E_{m}$-exposed mosquitoes

$I_{m}$-infectious mosquitoes

Some of the assumptions of our model include;

[i] Mosquito will die after infection

[ii] The rate at which humans and mosquito enter the population and die are respectively given by $\wedge_{h}, \wedge_{m}$ and $\alpha_{h}, \alpha_{m}$,

[iii] The rate at which human and mosquito die from disease induced deaths are respectively given as $\beta_{h}, \beta_{m}$

[iv] Individuals are allowed to move from susceptible human population to the exposed human population at a rate which is proportional to both size of susceptible human population and infected mosquito population and inversely proportional to total human population $\frac{\gamma S_{h} I_{m}}{N_{h}}$

$[v]$ Members of exposed class $\left(E_{h}\right)$ move to infected human class $\left(I_{h}\right)$ at a rate proportional to the number of individuals in the exposed class, $\rho E_{h}$,

[vi] Individuals in the infected class move to recovered class at a rate proportional to the number of individuals in the infected class, $\sigma I_{h}$.

[vii] Individuals in the recovered class move to susceptible class at a rate proportional to size of individuals in the recovered class, $\mu R_{h}$. 
[viii] For mosquito population, susceptible mosquitoes move to exposed class at a rate $\frac{\theta S_{m I_{h}}}{N_{h}}$

[ix] Mosquitoes in the exposed class move to infectious class at the rate proportional to the size of individuals in the exposed mosquito population $\omega E_{m}$

\subsection{Model Formulation}

HUMAN POPULATION

$\mu R_{h}$

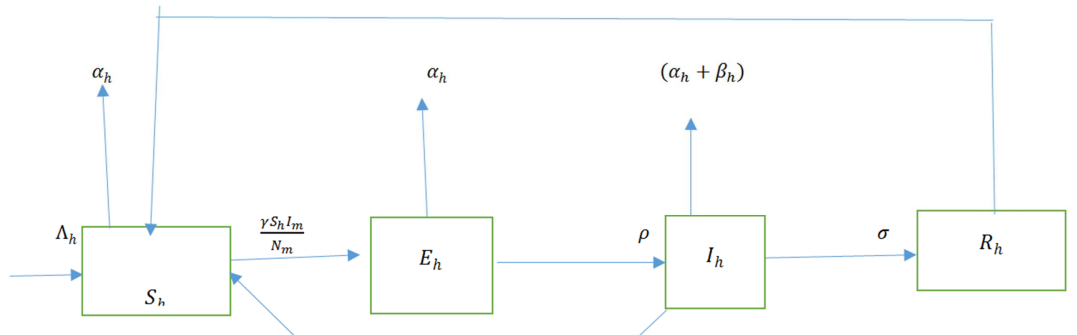

MOSQUITO POPULATION

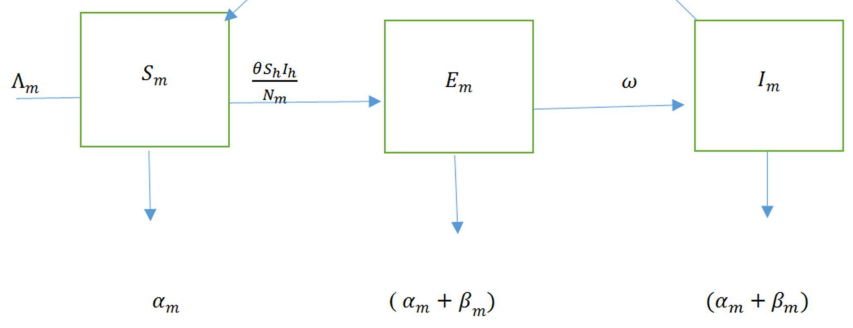

Figure 1. Human-mosquito flow diagram.

\subsection{Model Equations}

From the assumptions made, the following are equations from the model:

$$
\begin{gathered}
\frac{d s_{h}}{d t}=\Lambda_{h}+\mu R_{h}-\alpha_{h} S_{h}-\frac{\gamma S_{h} I_{m}(t-\tau)(1-x)}{N_{h}} \\
\frac{d E_{h}}{d t}=\frac{\gamma S_{h} I_{m}(t-\tau)(1-x)}{N_{h}}-\rho E_{h}(t-\tau)-\alpha_{h} E_{h}(t-\tau) \\
\frac{d I_{h}}{d t}=\rho E_{h}(t-\tau)-\left(\alpha_{h}+\beta_{h}\right) I_{h}-\sigma(1-z) I_{h}(t-\tau) \\
\frac{d R}{d t}=\sigma(1-z) I_{h}(t-\tau)-\alpha_{h} R_{h}-\mu R_{h} \\
\frac{d S_{m}}{d t}=-\frac{\theta I_{h}(t-\tau) S_{m}(1-x)}{N_{h}}+\Lambda_{m}-\left(\alpha_{m}+\beta_{m}+K x+L y\right) S_{m} \\
\frac{d E_{m}}{d t}=\frac{\theta S_{m} I_{h}(t-\tau)(1-x)}{N_{m}}-\omega E_{m}(t-\tau)-\left(\alpha_{m}+\beta_{m}+K x+L y\right) E_{m} \\
\frac{d I_{m}}{d t}=\omega E_{m}(t-\tau)-\left(\alpha_{m}+\beta_{m}+K x+L y\right) I_{m}
\end{gathered}
$$

\subsection{Positivity of Solutions}

The following theorem is used in determining positivity of our solutions.

Theorem

Let the initial data be

$$
\left\{\left(S_{h}(0), S_{m}(0) \geq 0,\left(E_{h}(0), I_{h}(0), R_{h}(0), E_{m}(0), I_{m}(0)\right\}\right.\right.
$$

Then the solution

$$
\left\{S_{h}, E_{h}, I_{h}, R_{h}, S_{m}, E_{m}, I_{m}\right\}(t)
$$


Of the system is non-negative for all $\mathrm{t} \geq 0$

Proof

From the system of equation (1) in the model

$$
\begin{gathered}
\frac{d S_{h}}{d t}=\Lambda_{h}+\mu R_{h}-\alpha_{h} S_{h}-\frac{\gamma S_{h} I_{m}(t-\tau)(1-x)}{N_{h}} \\
\geq-\alpha_{h} S_{h}-\frac{\gamma S_{h} I_{m}(t-\tau)(1-x)}{N_{h}} \\
\frac{d S_{h}}{d t} \geq-\left(\alpha_{h}+\frac{\gamma I_{m}(t-\tau)(1-x)}{N_{h}}\right) S_{h}
\end{gathered}
$$

Using separation of variables and integrating both sides

$$
\begin{gathered}
\int \frac{1}{S_{h}} d S_{h} \geq-\int\left(\alpha_{h}+\frac{\gamma I_{m}(t-\tau)(1-x)}{N_{h}}\right) d t \\
\ln S_{h} \geq-\left(\alpha_{h}+\frac{\gamma S_{h} I_{m}(t-\tau)(1-x)}{N_{h}}+c\right. \\
S_{h}(t)=e^{-\left(\alpha_{h}+\gamma I_{m}(t-\tau)(1-x) t \times e^{c}\right.}
\end{gathered}
$$

Let $e^{c}=K$

$$
\begin{aligned}
S_{h}(t)= & e^{-(} \alpha_{h}+\gamma I_{m}(t-\tau)(1-x) t \times K \\
& S_{h}(t)=K e^{-(} \alpha_{h}+\gamma I_{m}(t-\tau)(1-x) t
\end{aligned}
$$

When $t=0, S_{h}(0) \geq k$

$$
S_{h}(t) \geq S_{h}(0) e^{-(} \alpha_{h}+\gamma I_{m}(t-\tau)(1-x) t \geq 0
$$

From the second equation of system of equation (1)

$$
\begin{gathered}
\frac{d E_{h}}{d t}=\frac{\gamma S_{h} I_{m}(t-\tau)(1-x)}{N_{h}}-\rho E_{h}(t-\tau)-\alpha_{h} E_{h}(t-\tau) \\
\frac{d E_{h}}{d t} \geq-\left(\rho+\alpha_{h}\right) E_{h}(t-\tau)
\end{gathered}
$$

Integrating both sides we have

$$
\begin{aligned}
& \int \frac{1}{E_{h}} d E_{h} \geq-\int-\left(\rho+\alpha_{h}\right)(t-\tau) d t \\
& \ln E_{h} \geq-\left(\rho(t-\tau)+\alpha_{h}(t-\tau) t+c\right. \\
& E_{h}(t)=e^{-(} \rho(t-\tau)+\alpha_{h}(t-\tau) t \times e^{c}
\end{aligned}
$$

Let $e^{c}=K$

When $t=0, E_{h}(0) \geq e^{c}$

$$
\left.E_{h}(t) \geq E_{h}(0) e^{-(} \rho(t-\tau)+\alpha_{h}\right) t \geq 0
$$

Similarly, it can be shown that the remaining equations of the model are positive for all $t>0$, because $e^{\aleph}>0$, for all $\aleph \in \mathbb{R}$.

$$
V=\left[\begin{array}{cc}
\left(\rho-\alpha_{h}\right) e^{-\lambda \tau} & 0 \\
-\rho e^{-\lambda \tau} & \left(\alpha_{h}+\beta_{h}\right)+\sigma(1-z) e^{-\lambda \tau} \\
0 & 0 \\
0 & 0
\end{array}\right.
$$

$$
\omega e^{-\lambda \tau}+\left(\begin{array}{rcc}
0 & 0 & \\
0 & 0 & \\
0 & 0 \\
-\omega e^{-\lambda \tau} & & \left(\alpha_{m}+\beta_{m}+k x+l y\right)
\end{array}\right]
$$

Taking partial derivatives of

$$
\frac{\partial F_{i}}{\partial E_{h}}, \frac{\partial F_{i}}{\partial I_{h}}, \frac{\partial F_{i}}{\partial E_{m}}, \frac{\partial F_{i}}{\partial I_{m}}
$$

To get

$$
F=\left[\begin{array}{ccccc}
0 & 0 & 0 & \frac{\gamma S_{h}(1-x) e^{-\lambda \tau}}{N_{h}} \\
0 & 0 & & 0 & 0 \\
0 & \frac{\theta S_{m}(1-x) e^{-\lambda \tau}}{N_{m}} & & 0 & 0 \\
0 & 0 & & 0 & 0
\end{array}\right]
$$

Also from system of equation (1) we obtain $V_{i}$ as

$$
V_{i}=\left[\begin{array}{c}
\left(\rho-\alpha_{h}\right) E_{h}(t-\tau) \\
\left(\alpha_{h}+\beta_{h}\right) I_{h}+\sigma(1-z) I_{h}(t-\tau)-\rho E_{h}(t-\tau) \\
\omega E_{m}(t-\tau)+\left(\alpha_{m}+\beta_{m}+k x+l y\right) E_{m} \\
\left(\alpha_{m}+\beta_{m}+k x+l y\right) I_{m}-\omega E_{m}(t-\tau)
\end{array}\right]
$$

Taking partial derivatives of

$$
\frac{\partial V_{i}}{\partial E_{h}} \frac{\partial V_{i}}{\partial I_{h}} \frac{\partial V_{i}}{\partial E_{m}} \frac{\partial V_{i}}{\partial I_{m}}
$$

Let $A=\left(\rho-\alpha_{h}\right) e^{-\lambda \tau}$ 


$$
\begin{gathered}
B=-\rho e^{-\lambda \tau} \\
C=\left(\alpha_{h}+\beta_{h}\right)+\sigma(1-z) e^{-\lambda \tau} \\
D=\omega e^{-\lambda \tau}+\left(\alpha_{m}+\beta_{m}+k x+l y\right) \\
E=-\omega e^{-\lambda \tau} \\
F=\left(\alpha_{m}+\beta_{m}+k x+l y\right) \\
\text { Then, } V^{-1}=\left[\begin{array}{cccc}
\frac{1}{A} & 0 & 0 & 0 \\
\frac{-B}{A C} & \frac{1}{C} & 0 & 0 \\
0 & 0 & \frac{1}{D} & 0 \\
0 & 0 & \frac{-E}{D F} & \frac{1}{F}
\end{array}\right]
\end{gathered}
$$

And so,

$$
F V^{-1}=\left[\begin{array}{c}
0 \\
0 \\
\frac{-B \theta S_{m}\left((1-x) e^{-\lambda \tau}\right.}{N_{m} A C} \\
0
\end{array}\right.
$$

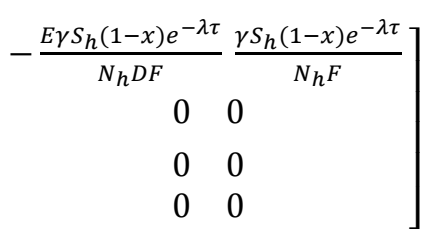

$$
\lambda^{2}=0 \text { or } \lambda^{2}=a c, \Rightarrow \lambda= \pm \sqrt{a c}
$$

$$
\begin{gathered}
\mathrm{a}=-\frac{E \gamma S_{h}(1-x) e^{-\lambda \tau}}{N_{h} D F} \\
\mathrm{~b}=\frac{\gamma S_{h}(1-x) e^{-\lambda \tau}}{N_{h} F} \\
\mathrm{c}=\frac{-B \theta S_{m}\left((1-x) e^{-\lambda \tau}\right.}{N_{m} A C} \\
\mathrm{~d}=\frac{\theta S_{m}\left((1-x) e^{-\lambda \tau}\right.}{N_{m} C}
\end{gathered}
$$

The dominant Eigen value or reproductive ratio is $\lambda=\sqrt{a c}$

Therefore,

$$
\begin{gathered}
R_{0}=\sqrt{\frac{\omega e^{-\lambda \tau} S_{h}(1-x) e^{-\lambda \tau}}{N_{h}\left(\left(\omega e^{-\lambda \tau}+\left(\alpha_{m}+\beta_{m}+k x+l y\right)\right)\left(\alpha_{m}+\beta_{m}+k x+l y\right)\right)}} \times \\
\sqrt{\frac{\rho e^{-\lambda \tau} \theta S_{m}\left((1-x) e^{-\lambda \tau}\right.}{\left.N_{m}\left(\left(\alpha_{h}+\beta_{h}\right)+\sigma(1-z) e^{-\lambda \tau}\right)\right)}}
\end{gathered}
$$

Then (13) becomes,

$$
F V^{-1}=\left[\begin{array}{cccc}
0 & 0 & a & b \\
0 & 0 & 0 & 0 \\
c & d & 0 & 0 \\
0 & 0 & 0 & 0
\end{array}\right]
$$

then the characteristic equation of (14) is given by,

$$
\left|F V^{-1}-\lambda I\right|=0
$$

Which implies that,

$$
\left|\begin{array}{cccc}
-\lambda & 0 & a & b \\
0 & -\lambda & 0 & 0 \\
c & d & -\lambda & 0 \\
0 & 0 & 0 & -\lambda
\end{array}\right|=0
$$

Therefore

$$
\begin{gathered}
-\lambda\left|\begin{array}{ccc}
-\lambda & 0 & a \\
0 & -\lambda & 0 \\
c & d & -\lambda
\end{array}\right|+a(0)-b(0)=0 \\
\lambda^{2}\left(\lambda^{2}-a c\right)=0
\end{gathered}
$$

\subsection{Existence of Disease Free Equilibrium}

In the absence of disease in the population we have;

$$
\left(E_{h}=I_{h}=, E_{m}=I_{m}=0=R_{h}\right) .
$$

Here, $R_{h}=0$ since there will be no disease to recover from, hence from the system of equation (1) we have,

$$
\begin{gathered}
\Lambda_{h}+\mu R_{h}-\alpha_{h} S_{h}-\frac{\gamma S_{h} I_{m}(t-\tau)(1-x)}{N_{h}}=0 \\
-\frac{\theta I_{h}(t-\tau) S_{m}(1-x)}{N_{h}}+\Lambda_{m}-\left(\alpha_{m}+K x+L y\right) S_{m}=0
\end{gathered}
$$

Since $I_{m}=0$ in (19)

We have,

$$
S_{h}=\frac{\Lambda_{h}}{\alpha_{h}}
$$

Since $I_{h}=0$ in (20) then,

$$
\frac{\Lambda_{m}}{\alpha_{m}+k x+l y}=S_{m}
$$

Similarly, when $\left(E_{h}=I_{h}=, E_{m}=I_{m}=0=R_{h}\right)$ in the 
remaining equations becomes

$$
E_{h}^{0}=0,, I_{h}^{0}=0, R_{h}^{0}=0,, S_{m}^{0}=\frac{\Lambda_{m}}{\alpha_{m}+k x+l y}, E_{m}^{0}=0, I_{m}^{0}=0
$$

Where $\mathrm{E}_{\mathrm{h}}^{0}$, -at disease free equilibrium and likewise $\mathrm{I}_{\mathrm{h}}^{0}, \mathrm{R}_{\mathrm{h}}^{0}, \mathrm{~S}_{\mathrm{m}}^{0}, \mathrm{E}_{\mathrm{m}}^{0}$ and, $\mathrm{I}_{\mathrm{m}}^{0}$ respectively

Therefore disease free equilibrium point of our malaria model is given by

$$
E_{0}=\left(S_{h}^{0}, E_{h}^{0}, I_{h}^{0}, R_{h}^{0}, S_{m}^{0}, E_{m}^{0}, I_{m}^{0}\right)=\left(\frac{\Lambda_{h}}{\alpha_{h}}, 0,0,0 \frac{\Lambda_{m}}{\alpha_{m}+k x+l y}, 0,0\right)
$$

$$
\boldsymbol{J}=\left[\begin{array}{cccc}
-\alpha_{h} & 0 & 0 & \mu \\
0 & -\rho e^{-\lambda \tau}-\alpha_{h} e^{-\lambda \tau} & 0 & 0 \\
0 & \rho e^{-\lambda \tau} & -\left(\alpha_{h}+\beta_{h}\right)-\sigma(1-z) e^{-\lambda \tau} & 0 \\
0 & 0 & \sigma(1-z) e^{-\lambda \tau} & -\alpha_{h}-\mu \\
0 & 0 & -\frac{\theta s_{m}(1-x) e^{-\lambda \tau}}{\left(\alpha_{m}+k x+l y\right) N_{m}} & 0 \\
0 & 0 & \frac{\theta s_{m}(1-x) e^{-\lambda \tau}}{\left(\alpha_{m}+k x+l y\right) N_{m}} & 0 \\
0 & 0 & 0 & 0
\end{array}\right.
$$

The system of equation [1] is stable if all the Eigen values of linearization matrix are negative.

In solving the eigen values we let,

$$
\begin{gathered}
\mathrm{a}=-\alpha_{h}, \mathrm{~b}=-\gamma \frac{s_{h}(1-x) e^{-\lambda \tau}}{N_{h}}, \mathrm{c}=-\rho e^{-\lambda \tau}-\alpha_{h} e^{-\lambda \tau}, \\
\mathrm{d}=\gamma \frac{s_{h}(1-x) e^{-\lambda \tau}}{N_{h}}, \mathrm{e}=\rho e^{-\lambda \tau} \\
\mathrm{f}=-\left(\alpha_{h}+\beta_{h}\right)-\sigma(1-z) e^{-\lambda \tau}, \mathrm{g}=\sigma(1-z) e^{-\lambda \tau}, \mathrm{h}=-\alpha_{h}-\mu, \\
\mathrm{i}=-\frac{\theta s_{m}(1-x) e^{-\lambda \tau}}{N_{m}}, \mathrm{j}=-\alpha_{m}-\beta_{m}-k x-l y \\
\mathrm{k}=\frac{\theta s_{m}(1-x) e^{-\lambda \tau}}{N_{m}}, \mathrm{l}=-\omega e^{-\lambda \tau}-\alpha_{m}-\beta_{m}-k x-l y, \mathrm{~m}=\omega e^{-\lambda \tau}, \\
\mathrm{n}=-\alpha_{m}-\beta_{m}-k x-l y
\end{gathered}
$$

Solving the eigen values of the jacobian matrix $|J-\lambda I|=0$

$$
\left[\begin{array}{cccc}
c+\lambda & 0 & 0 & d \\
e & f+\lambda & 0 & 0 \\
c & k & l+\lambda & 0 \\
0 & 0 & m & n+\lambda
\end{array}\right]=0
$$

We have

$$
(c+\lambda)(n+\lambda)(f+\lambda)(l+\lambda)-d m e k=0
$$

To simplify the equation (25)

Let

$$
A_{1}=n, A_{2}=l, A_{3}=f, A_{4}=c \text { and } Q=\text { dmek }
$$

This implies

$$
\begin{gathered}
\left(\lambda+A_{1}\right)\left(\lambda+A_{2}\right)\left(\lambda+A_{3}\right)\left(\lambda+A_{4}\right)-Q=0 \\
\lambda^{4}+\lambda^{3} B_{1}+\lambda^{2} B_{2}+\lambda B_{3}+B_{4}=0
\end{gathered}
$$

Where,

$$
B_{1}=A_{4}+A_{3}+A_{2}+A_{1}
$$

This is the state where there is no malaria in the population.

\subsection{Stability of Disease Free Equilibrium}

The stability of the disease free equilibrium state can be tested using Eigen values of a jacobian matrix obtained at DFE, this is where $R_{O}<1$. The linearization matrix of system of equation (1) at disease free equilibrium is given by

$$
\left.\begin{array}{ccc}
0 & 0 & -\gamma \frac{s_{h}(1-x) e^{-\lambda \tau}}{N_{h}} \\
0 & 0 & \gamma \frac{s_{h}(1-x) e^{-\lambda \tau}}{N_{h}} \\
0 & 0 & 0 \\
0 & 0 & 0 \\
-\alpha_{m}-\beta_{m}-k x-l y & 0 & 0 \\
0 & -\omega e^{-\lambda \tau}-\alpha_{m}-\beta_{m}-k x-l y & 0 \\
0 & \omega e^{-\lambda \tau} & -\alpha_{m}-\beta_{m}-k x-l y
\end{array}\right]
$$

Therefore $R_{0}$ in equation (18) can be written in terms of $A_{i}$ where $i=1,2,3,----n$

As

$$
R_{0}^{2}=\frac{\left(\omega e^{-\lambda \tau} \theta\left((1-x) e^{-\lambda \tau} S_{m}\right)\left(\rho e^{-\lambda \tau}(1-x) e^{-\lambda \tau} S_{h}\right)\right.}{N_{m} N_{h} A_{3} A_{2} A_{1}^{2}}
$$

Using the Routh-Hurwitz criteria on equation (27) we can show that all roots have negative real parts.

Routh -Hurwitz criteria[15] gives necessary and sufficient conditions for all the roots of characteristic polynomial and lies on the left half of the complex plane.

Theorem 2.8 Routh-Hurwitz criteria.

Given the polynomial

$$
P(\lambda)=\lambda^{n}+\lambda^{n-1} B_{1}+--+\lambda B_{n-1}+B_{n}
$$

Where the coefficients $B_{i}$ are real constants $i=1,---n$, define the $n$ Hurwitz matrices using the coefficients $B_{i}$ of the characteristic polynomial

Where $H_{1}=\left(B_{1}\right), H_{2}=\left[\begin{array}{cc}B_{1} & 1 \\ B_{3} & B_{2}\end{array}\right], H_{3}=\left[\begin{array}{ccc}B_{1} & 1 & 0 \\ B_{3} & B_{2} & B_{1} \\ B_{5} & B_{4} & B_{3}\end{array}\right]$,

$H_{n}=\left[\begin{array}{ccccccc}B_{1} & 1 & 0 & 0 & 0 & \prime & 0 \\ B_{3} & B_{2} & B_{1} & 1 & 0 & \prime & 0 \\ B_{5} & B_{4} & B_{3} & B_{2} & B_{1} & , & 0 \\ \prime & \prime & \prime & \prime & \prime & \prime & 0 \\ \prime & \prime & \prime & \prime & \prime & \prime & 0 \\ \prime & \prime & \prime & \prime & \prime & , & 0 \\ 0 & 0 & 0 & 0 & 0 & \prime & B_{n}\end{array}\right]$

Where $B_{\mathrm{j}}=0$ if $j>n$, all the roots of the polynomial $P(\lambda)$ are negative if and only if the determinants of Hurwitz matrices are positive 


$$
\operatorname{det}\left(H_{\mathrm{j}}\right)>0, j=1,2---n
$$

For the equation (27), when $\mathrm{n}=4$, the Routh-Hurwitz criteria are

$B_{1}>0, B_{2}>0, B_{3}>0, B_{3}>0$ and the determinants of Hurwitz matrices are:

$$
\begin{gathered}
\operatorname{det}\left(H_{1}\right)=B_{1}>0 \\
\operatorname{det}\left(H_{2}\right)=\left[\begin{array}{cc}
B_{1} & 1 \\
0 & B_{2}
\end{array}\right], B_{1} B_{2}>0 \\
\operatorname{det}\left(H_{3}\right)=\left[\begin{array}{ccc}
B_{1} & 1 & 0 \\
B_{3} & B_{2} & B_{1} \\
0 & 0 & B_{3}
\end{array}\right]=0, B_{1} B_{2} B_{3}-B_{3}^{2}>0 \\
\operatorname{det}\left(H_{4}\right)=\left[\begin{array}{cccc}
B_{1} & 1 & 0 & 0 \\
B_{3} & B_{2} & B_{1} & 1 \\
0 & B_{4} & B_{3} & B_{2} \\
0 & 0 & 0 & B_{4}
\end{array}\right] \\
=0, B_{1}\left(B_{2} B_{1}-B_{3}\right)-B_{4} B_{1}^{2}>0
\end{gathered}
$$

Clearly, from Hurwitz matrices all the determinants are positive, which means that all the Eigen values of the jacobian matrix have negative real part. Moreover, if $R_{0}<1$, it follows that from (28) that $A_{i}>0$ and therefore disease free equilibrium point is stable when $R_{0}<1$.

\section{Numerical Simulations and Results}

The simulations were performed using MATLAB'S built in dde 23 solver. In the analysis, initial population sizes and other parameters were obtained from literature as shown from the table below.

Table 1. Description of variables and parameters of malaria model.

\begin{tabular}{lll}
\hline PARAMETER & VALUE & REFERENCE \\
\hline$\Lambda_{h}$ & 0.028 per day & Chiyaka et al (2008) \\
$\Lambda_{m}$ & 6 & Estimated \\
$\rho$ & $1 / 14$ & Malaria.com (2011) \\
$\alpha_{h}$ & 0.00004 per day & Hyun $(2001)$ \\
$\beta_{h}$ & 0.0004 per day & Prince Harvim (2014) \\
$\beta_{m}$ & 0.01 per day & Chiyaka et al (2008) \\
$\alpha_{m}$ & 0.04 per day & Chiyaka et al $(2008)$ \\
$\mathrm{Z}$ & 0.2 per day & Estimated \\
$\mathrm{K}$ & $1 / 365$ & Estimated \\
$\mathrm{L}$ & $1 / 365$ & Estimated \\
$\gamma$ & 0.0025 & Estimated \\
$\mu$ & 0.04 & Estimated \\
$\omega$ & $1 / 12$ & Chiyaka etal (2008) \\
$\theta$ & 0.0415 & Estimated \\
$\sigma$ & 0.005 & Estimated \\
$\lambda$ & 0.06 & Estimated \\
$x$ & 0.5 & Estimated \\
$y$ & 0.5 & Estimated \\
\hline
\end{tabular}

The initial conditions used are:

$$
\begin{gathered}
S_{h}=300, E_{h}=200, I_{h}=100, R_{h}=50, S_{m}=400, E_{m}= \\
300, I_{m}=200 \mathrm{t}=350 \text { days }
\end{gathered}
$$

Numerical simulation.

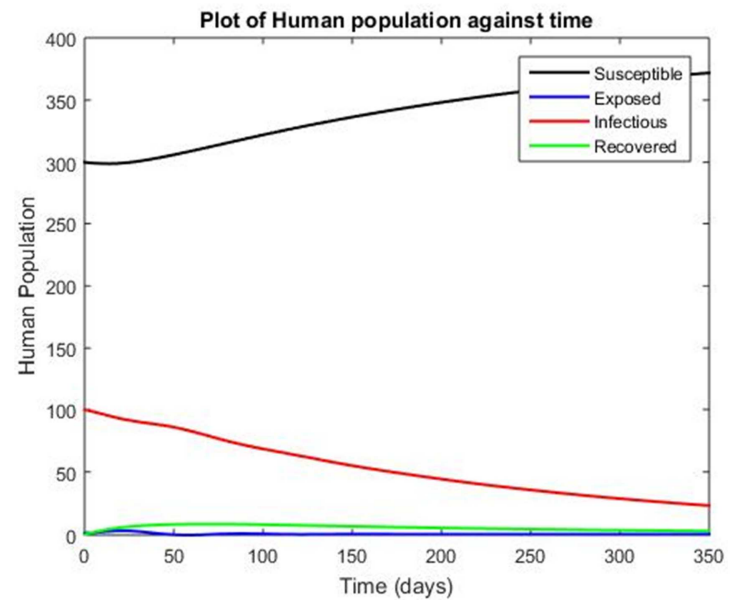

Figure 2. Shows human population against time in days.

From the graph it shows that the number of infective humans reduces considerably because of the use of control strategies, while the number of humans susceptible also increases because the disease is under control.

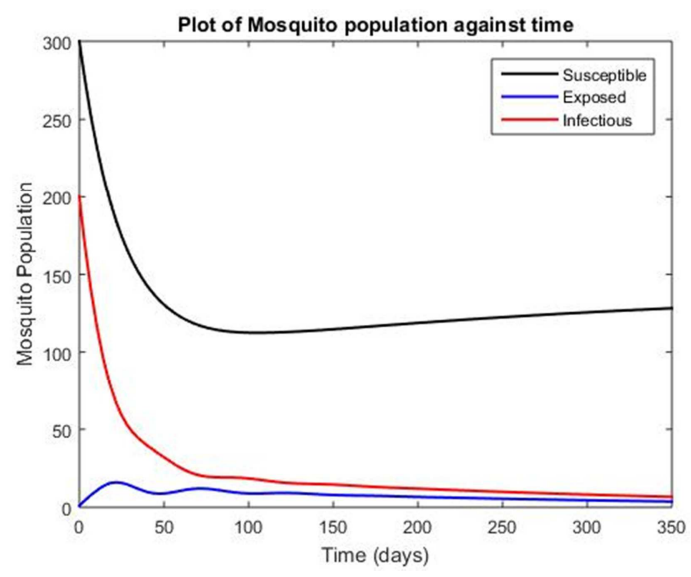

Figure 3. Shows mosquito population with time.

From the figure it is evident that the number of infective mosquitoes went down significantly with time as a result of a combination of control strategies and treatment used, while those exposed to disease dropped significantly.

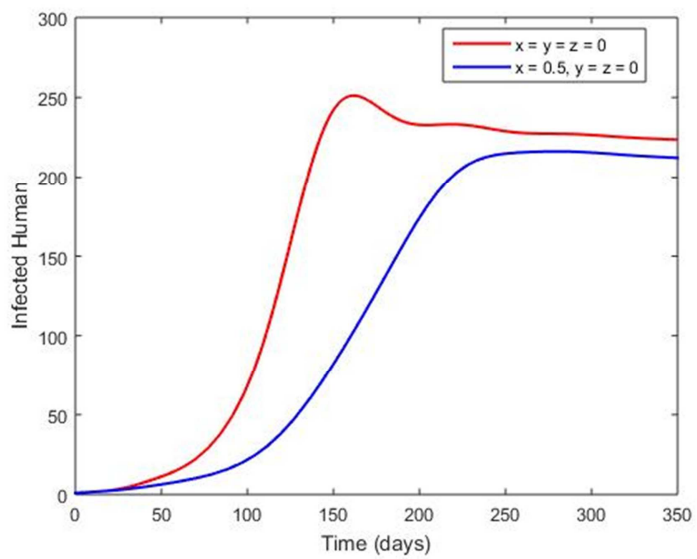




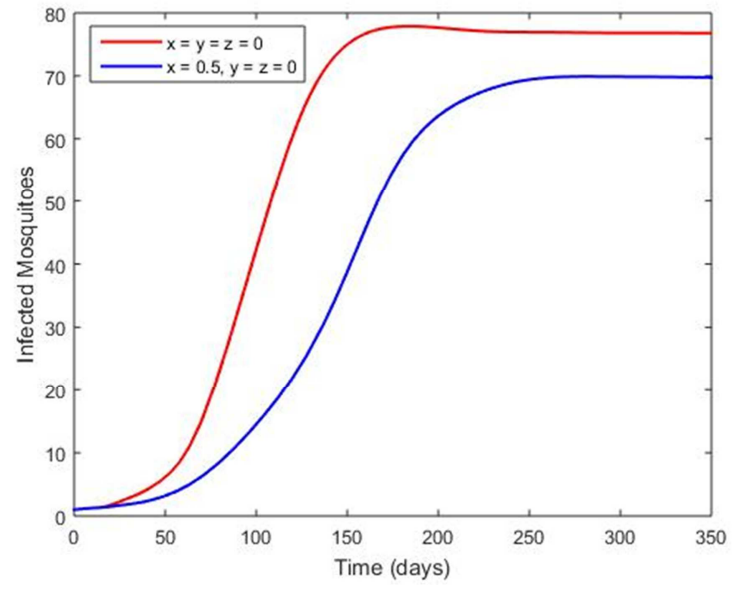

Figure 4. Simulation of Long Lasting Insecticides Treated Nets (LLINS).

From figure 4 . above when the contol strategies are not used $\mathrm{x}=\mathrm{y}=\mathrm{z}=0$ the number of infective humans was high, and when only LLINS (x) was used the number of infective humans and mosquitoes dropped.
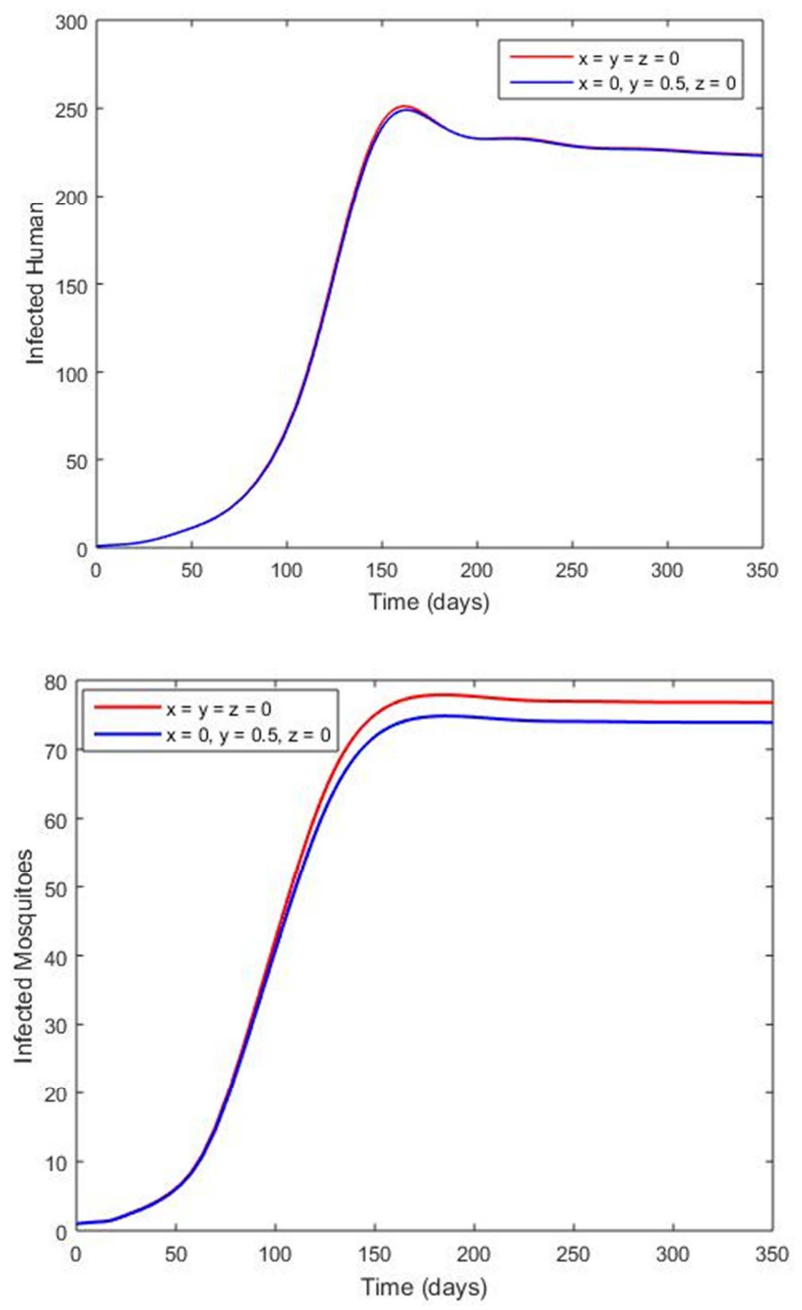

Figure 5. Simulation of Indoor Residual Spraying (IRS).

Figure 5 From the above figure it is evident that indoor residual spraying reduced the number of mosquitoes, while it didn't have any impact on infected human population
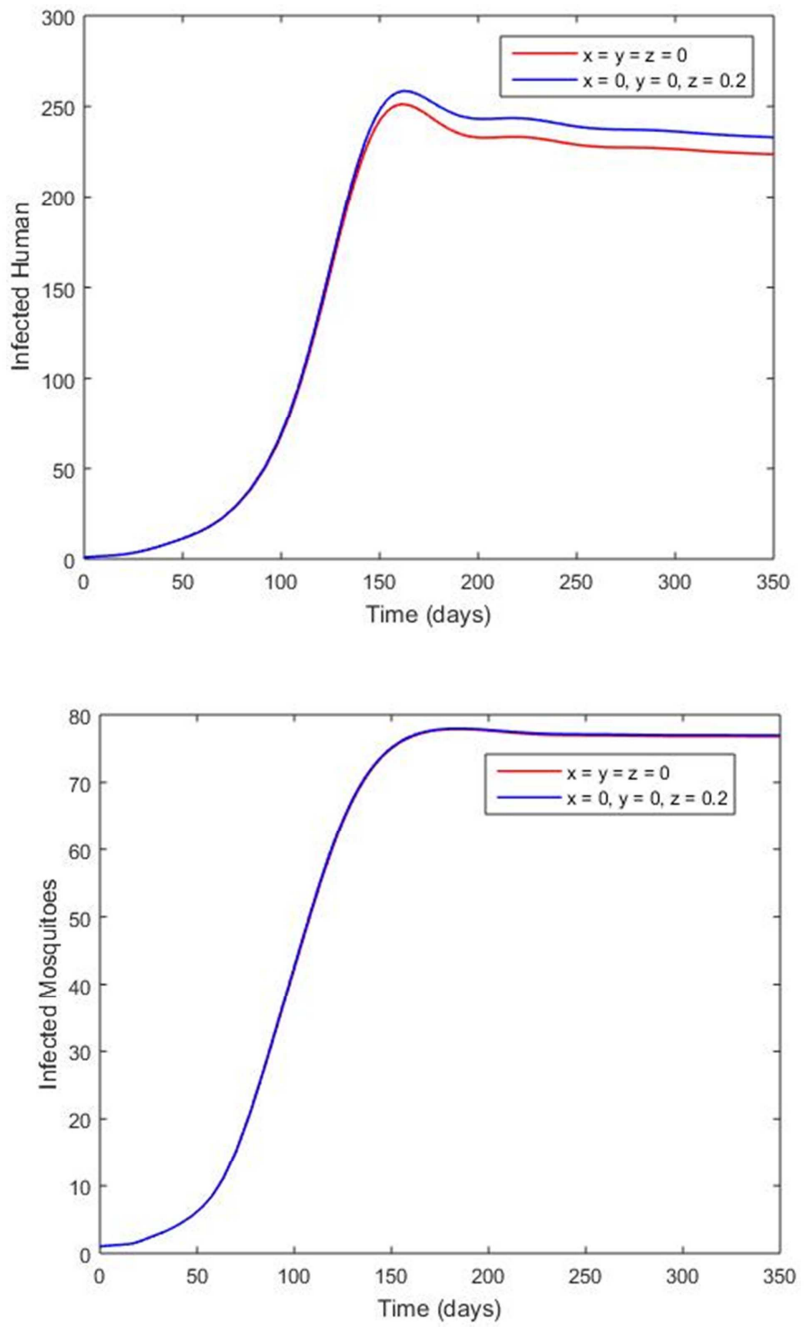

Figure 6. Simulation ofTreatment (with drug).

From figure 6. It shows that with the use of drugs for treatment of malaria, it reduced the number of infected humans with time.

\section{Conclusion}

From the study, SEIR and SEI model for humans and mosquitoes were used to study malaria transmission dynamics. The model is achieved with control strategies such as; use of long lasting treated bed nets (LLINS), indoor residual spraying, intermittent preventive treatment for infants and pregnant mothers.

The model equations generated were used to calculate reproduction number using the next generation matrix. From the results it was found out that when $R_{0}<1$ the model was locally stable and the disease was controlled and when

$R_{0}>1$, the disease persists because number of infective humans increased.

Therefore with the combination of control strategies and treatment, the malaria spread is put on control. 


\section{Future Research and Suggestions}

The model in our research has not exhausted all the strategies, like developing a malaria vaccine to check on malaria spread. Future model should be developed to include the effects of environment on the number of infective mosquitoes.

\section{References}

[1] Nyangera, O. W. (2013). A Mathematical model for the control of malaria with temporary immunity. Thesis paper University of Nairobi.

[2] WHO (2016). World malaria report. Retrieved from http://www.who.int/malaria/publications.

[3] Diekmann o, H. J. (2000). Mathematical epidemiology of infectious diseases. model builing, analysis and interpretation, 503-522.

[4] Tumwiine, J. e. (2014). A mathematical model for transmission and spread of drug sensitive and resistant malaria strains within a human population. ID 636973.

[5] Sunita, D. e. (2017). A SEIR model for malaria with infective immigrant. International Journal of Recent Research Vol 11, No 2, pg 155-160.

[6] Abay, A. (2015). Mathematical modelling of endemic malaria transmission. America Journal of Applied Mathematics, 3646.
[7] Jessicca, M. e., \& etal. (2018). Qualitative analysis of a mathematical model applied to malaria transmission in Tumaco.

[8] Oduro, F. a. (2012). Transmission dynamics of malaria in Ghana. Journal of mathematics research, 4:6.

[9] Mojeeb, K. A. (2017). A Simple SEIR mathematical model of malaria transmission. Asian Research Journalof Mathematics.

[10] Ephraim Agyingi, M. N. (2016). The dynamics of multiple species and strains of malaria. School of mathematical sciences, Rochester Institute of technology vol 1, 29-40.

[11] Prince Harvim (2014), An epidemiological model of malaria transmission in Ghana. Thesis paper Kwame Nkurumah University and Technology.

[12] Nisha Budhar, S. D. (2017). Stability analysis of Humanmosquito model of malaria. International journal of mathematical and computational sciences Vol 11.

[13] Kbenesh, B. e. (2009). 0ptimal control of vector-borne diseases: treatment and prevention. Discrete continuous dynamical system B11 (3), 587-611.

[14] https./dph.georgia.gov.(2014). Malaria Georgia department of public health.

[15] Flores J. D. Math-735. Mathematical modelling. 4.5 RouthHurwitz criteria. Department of mathematical sciences. The University of south Dakota, jFlores@usd.edu. 